Article

\title{
Synthesis, Physicochemical Characterization, and Cytotoxicity Assessment of Rh Nanoparticles with Different Morphologies-as Potential XFCT Nanoprobes
}

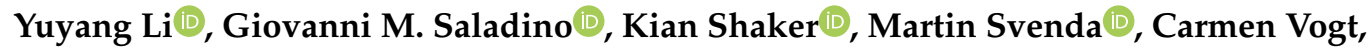 \\ Bertha Brodin, Hans M. Hertz and Muhammet S. Toprak * (D) \\ Biomedical and X-ray Physics, Department of Applied Physics, KTH Royal Institute of Technology, \\ SE 10691 Stockholm, Sweden; yuyangli@kth.se (Y.L.); saladino@kth.se (G.M.S.); kiansd@kth.se (K.S.); \\ martin.svenda@biox.kth.se (M.S.); carmenma@kth.se (C.V.); berthab@kth.se (B.B.); \\ hans.hertz@biox.kth.se (H.M.H.) \\ * Correspondence: toprak@kth.se; Tel.: +46-735-519-358
}

Received: 25 September 2020; Accepted: 23 October 2020; Published: 27 October 2020

check for updates

\begin{abstract}
Morphologically controllable synthesis of Rh nanoparticles (NPs) was achieved by the use of additives during polyol synthesis. The effect of salts and surfactant additives including PVP, sodium acetate, sodium citrate, CTAB, CTAC, and potassium bromide on Rh NPs morphology was investigated. When PVP was used as the only additive, trigonal NPs were obtained. Additives containing $\mathrm{Br}^{-}$ions (CTAB and $\mathrm{KBr}$ ) resulted in NPs with a cubic morphology, while those with carboxyl groups (sodium citrate and acetate) formed spheroid NPs. The use of $\mathrm{Cl}^{-}$ions (CTAC) resulted in a mixture of polygon morphologies. Cytotoxicity of these NPs was evaluated on macrophages and ovarian cancer cell lines. Membrane integrity and cellular activity are both influenced to a similar extent, for both the cell lines, with respect to the morphology of Rh NPs. The cells exposed to trigonal Rh NPs showed the highest viability, among the NP series. Particles with a mixed polygon morphology had the highest cytotoxic impact, followed by cubic and spherical NPs. The Rh NPs were further demonstrated as contrast agents for X-ray fluorescence computed tomography (XFCT) in a small-animal imaging setting. This work provides a detailed route for the synthesis, morphology control, and characterization of Rh NPs as viable contrast agents for XFCT bio-imaging.
\end{abstract}

Keywords: polyol synthesis; rhodium nanoparticles; surfactants; role of additives; morphology control; toxicity; bio-imaging; $\mathrm{X}$-ray fluorescence; contrast agent; XFCT

\section{Introduction}

Like noble metals Pt and Pd, Rh nanomaterials have caught increasing attention due to applications in catalysis, photonics, and biosensors [1-4]. Physical and chemical properties of nanomaterials are strongly related to the size and morphology of particles. For instance, catalytic properties are associated with the active sites, which is reflected by the spatial shape and exposed crystal facets of metallic nanoparticles (NPs) [5]. In general, a high specific surface area contributes to a high catalytic activity, due to high-index crystal planes showing high binding affinity $[5,6]$. Likewise, nanomaterials also reveal an inherent correlation between toxicity and their surface properties within biological applications [7-10]. The specific surface-exposed groups could be the reactive sites inducing superoxide radical formation, as major reactive oxygen species (ROS) which are cytotoxic [10]. The hydrophilicity-hydrophobicity, or lipophilicity-lipophobicity, of nanomaterials functionalized by various groups also plays a key role in the toxicity and biocompatibility. Metallic nanomaterials, including $\mathrm{Ag}$ and $\mathrm{Au}$, show biocompatibility 
with a strong dependence on particle size, morphology, and surface properties [11]. The toxicity of Rh NPs as a function of their particle morphology has been rarely discussed in the literature [12]. It is, therefore, scientifically important to explore Rh NPs' morphological effects in connection with their potential biological applications.

Enormous efforts have been devoted to tune the synthesis methods in order to control the morphology and surface properties of metallic NPs. Surface capping agents such as salts and surfactants have shown significant effects on directing the growth of nanocrystals. A diverse set of morphologies of Ag and Au NPs have been reported using different capping agents. For example, PVP preferentially adsorbs on and reduces the growth rate of the $\mathrm{Ag}(100)$ surface, while PVP passivates the $\mathrm{Au}(111)$ surface. Halides anions like $\mathrm{Br}^{-}$also adsorb on the $\mathrm{Pd}(100)$ surface. Furthermore, citrate is able to passivate the $\mathrm{Pd}(111)$ surface [8]. Yu et al. [13] reported on the surface energy of noble metals $\mathrm{Rh}, \mathrm{Pt}$, $\mathrm{Pd}$, and $\mathrm{Au}$ that is different for various nanocrystal shapes, where $\mathrm{Rh}$ showed the highest surface energy for the crystal planes with the same index. The difference in surface energy values is significant (about five-six times higher) when compared to $\mathrm{Au}$, which makes $\mathrm{Rh}$ unique as a catalyst. However, if necessary, it is possible to passivate the catalytic activity with selective surface-adsorbed ligands. Furthermore, this extraordinarily high surface energy makes it rather challenging to have a good shape control for Rh [13]. There are very few studies, though, focusing on the morphology control of Rh particles. $\mathrm{Br}^{-}$ions, for instance, were reported to passivate the (100) plane in $\mathrm{Rh}$ nanocrystals [14]. The morphology control of metallic Rh NPs during typical polyol synthesis was demonstrated by replacing the Rh salt precursors with organometallic $\mathrm{Rh} \mathrm{Rh}_{2}\left(\mathrm{COOCF}_{3}\right)_{4}$, and the solvent with other polyols such as diethylene glycol, or tri-ethylene glycol [1,15].

We recently demonstrated the potential use of Rh NPs as X-ray fluorescence computed tomography (XFCT) contrast agents [16,17]. CCK-8-based cytotoxicity assays of triangular Rh NPs based on two cells lines, murine macrophages and human-derived ovarian cancer cells, showed toxicity for doses above $40 \mathrm{mg} / \mathrm{L}$ [17]. Further research is needed to understand the toxicity induced by Rh NPs. In this work, we present a systematic study on the effect of halide ions and carboxyl salts on the morphology of Rh NPs by one-pot polyol synthesis. The morphological and surface properties and cytotoxicity of morphologically different Rh NPs are studied and discussed, finally demonstrating the performance of Rh NPs in a small-animal XFCT imaging scenario.

\section{Materials and Methods}

\subsection{Materials}

Rhodium (III) chloride hydrate $\left(\mathrm{RhCl}_{3} \cdot \mathrm{xH}_{2} \mathrm{O}, \mathrm{Rh} 38.5-45.5 \%\right)$, Ethylene glycol (EG, $\mathrm{HOCH}_{2} \mathrm{CH}_{2} \mathrm{OH},>99 \%$ ), Poly(vinyl pyrrolidone) (PVP, $\mathrm{C}_{2} \mathrm{H}_{2} \mathrm{~N}\left(\mathrm{C}_{6} \mathrm{H}_{9} \mathrm{NO}\right)_{n} \mathrm{C}_{13} \mathrm{H}_{10} \mathrm{NS}_{2}$, average $\mathrm{MW}=55 \mathrm{kDa})$, Potassium bromide $(\mathrm{KBr},>99 \%)$, Sodium Acetate trihydrate (NaAc; $\left.\mathrm{CH}_{3} \mathrm{COONa} \cdot 3 \mathrm{H}_{2} \mathrm{O},>99 \%\right)$, Hexadecyltrimethylammonium bromide $\left(\mathrm{CTAB}, \mathrm{CH}_{3}\left(\mathrm{CH}_{2}\right)_{15} \mathrm{~N}(\mathrm{Br})\left(\mathrm{CH}_{3}\right)_{3}\right.$, $>99 \%$ ), Hexadecyltrimethylammonium chloride (CTAC, $\left.\mathrm{CH}_{3}\left(\mathrm{CH}_{2}\right)_{15} \mathrm{~N}(\mathrm{Cl})\left(\mathrm{CH}_{3}\right)_{3},>99 \%\right)$, and Sodium citrate dihydrate $\left(\mathrm{NaCit} ; \mathrm{HOC}(\mathrm{COONa})\left(\mathrm{CH}_{2} \mathrm{COONa}\right)_{2} \cdot 2 \mathrm{H}_{2} \mathrm{O}, 99 \%\right)$ were obtained from Sigma Aldrich, Germany. Hydrochloric acid, Sodium hydroxide, and solvents, including acetone and ethanol, were of analytical grade and were obtained from Sigma Aldrich, Sweden. All chemicals were used without further purification.

\subsection{Synthesis of Rh NPs}

The synthesis was based on a modified polyol reduction process [17]. In a typical synthesis, $20 \mathrm{~mL}$ EG is used as solvent. The halide salts, surfactants (molar ratio of Rh precursor/additive 1:1), and PVP (4 mmol in repeating units) were dissolved in EG in a glycerol bath. A series of reactions were performed for tuning the morphology of Rh NPs, by using the following additives in combination with PVP: Na-Ac, Na-Cit, CTAB, KBr, and CTAC. Details of all the synthesized samples are given in Table 1 . After the addition of $0.2 \mathrm{mmol} \mathrm{RhCl}_{3}$, the solution was heated to $85^{\circ} \mathrm{C}$, where nucleation was initiated, 
monitored from the darkening of the solution's color. After $15 \mathrm{~min}$, the temperature was ramped up to $115^{\circ} \mathrm{C}$, where it was kept for $2 \mathrm{~h}$, followed by quenching. The NPs obtained were centrifuged and washed three times through precipitation with acetone and re-dispersion in deionized water (DIW). Thereafter, they were dispersed in DIW for further characterizations.

Table 1. Summary of Rh nanoparticles (NPs) with diverse morphologies, synthesized using various additives.

\begin{tabular}{cccccc}
\hline Sample Designation & Precursor & Solvent & Additive & Stabilizer & Morphology \\
\hline Rh_PVP & $\mathrm{RhCl}_{3}$ & EG & - & PVP & triangle \\
Rh_PVP-KBr & $\mathrm{RhCl}_{3}$ & EG & $\mathrm{KBr}$ & PVP & cubic \\
Rh_PVP-CTAB & $\mathrm{RhCl}_{3}$ & EG & CTAB & PVP & cubic \\
Rh_PVP-CTAC & $\mathrm{RhCl}_{3}$ & EG & CTAC & PVP & polygon \\
Rh_PVP-NaAc & $\mathrm{RhCl}_{3}$ & EG & $\mathrm{NaAc}$ & PVP & spherical \\
Rh_PVP-NaCit & $\mathrm{RhCl}_{3}$ & EG & NaCit & PVP & spherical \\
\hline
\end{tabular}

\subsection{Characterization Methods}

The morphology and crystallinity of Rh samples were characterized by transmission electron microscopy (TEM) (JEM-2100F, 200 kV, JEOL Ltd., Tokyo, Japan). The TEM samples were prepared by transferring a $\sim 10 \mu \mathrm{L}$ droplet of colloidal suspension onto a carbon-coated copper grid (Ted-Pella, CA, USA), and drying for $12 \mathrm{~h}$. Particle size was measured by ImageJ by counting around 200 NPs in different fields of view on several TEM micrographs. Dynamic light scattering (DLS, Malvern Nano-ZS90, Malvern, UK) was used to measure the hydrodynamic size distribution of the colloidal suspension of as-prepared Rh NPs dispersed in DI water, at pH 7. Surface charge (Zeta potential) was also measured on the same samples using the same system under AC field. Inductively coupled plasma-optical emission spectroscopy (ICP-OES) (iCAP 6000 series, Thermo Scientific, Waltham, MA, USA) was used for the determination of the elemental Rh concentration in the colloidal suspensions prior to cytotoxicity tests. Ultraviolet-visible spectroscopy (UV-Vis) (NanoPhotometer NP80, Implen, Munich, Germany) was used to measure the absorption spectroscopy of Rh samples synthesized with different additives. Fourier-transform infrared spectroscopy (FT-IR, Thermo Scientific Nicolet iS20, Stockholm, Sweden) was used to obtain FT-IR spectra in transmission mode, on KBr pellets (2 mg sample in $100 \mathrm{mg} \mathrm{KBr}$ ), in the spectral range of $4000-400 \mathrm{~cm}^{-1}$. Thermal gravimetric analysis (TGA) was performed on dried samples in the temperature range of $30-700{ }^{\circ} \mathrm{C}$, using the TGA 550 system (TA instruments, Sollentuna, Sweden).

\subsection{In Vitro Toxicity}

Before proceeding with the in vitro toxicity analysis, Rh NP suspensions were tested for lipopolysaccharides (LPS) contamination [18] with PTS cartridges with a sensitivity of $0.005 \mathrm{EU} / \mathrm{mL}$ (Endosafe-PTS ${ }^{\mathrm{TM}}$, Charles River) following the manufacturer's instructions. All the NPs suspensions had LPS values below the maximum admissible limit of $0.1 \mathrm{EU} / \mathrm{mL}$ [19].

Toxicity tests were performed on two different cell lines which were RAW 264.7 (murine macrophages, 91062702-1VL, SigmaAldrich, Stockholm, Sweden) and SKOV-3-human-derived ovarian cancer (ATCC HTB-77, Wesel, Germany). Two different toxicity assays were used which were Cell Counting Kit-8 (CCK-8, Cat.\# 96922, Sigma Aldrich, Stockholm, Sweden) and NucGreen ${ }^{\circledR}$ viability assay (NucGreen ${ }^{\mathrm{TM}}$ Dead 488 ReadyProbes $^{\mathrm{TM}}$, Cat\# R37109, Thermo Fisher, Stockholm, Sweden). All the tests were performed following the supplier's instructions; the absorbance (CCK8) and fluorescence (NucGreen ${ }^{\circledR}$ ) measurements were performed on a Hidex Sense multi plate reader (Kem-En-Tec Nordic, Uppsala, Sweden). The viability of the cells exposed to NPs is normalized to the viability of the non-exposed cells (the negative control). 
Dulbecco's modified Eagle medium (DMEM, Sigma Aldrich) containing 10\% fetal bovine serum (FBS) was used as cell culture medium. As negative control, cells grown in the absence of NPs were used. The cells were about $80 \%$ confluent when the assays were performed.

The cells were split and seeded into 96-well plates (Cat. \# 167008, Thermo Fisher, Stockholm, Sweden), corresponding to 30,000 to 35,000 cells per well in a 96-well plate, one day before NP exposure. The cells were exposed for $24 \mathrm{~h}$ to a dilution series of Rh NPs with different morphologies. The concentration of NPs tested was highest at $250 \mu \mathrm{g} / \mathrm{mL}$ in the first well, and further dilution series were obtained by a 2 -fold dilution (as $125,62.5$, and $31.25 \mu \mathrm{g} / \mathrm{mL}$ ) of the stock to obtain a concentration series. Only in the case of the Rh_PVP-CTAC sample, the highest concentration was $100 \mu \mathrm{g} / \mathrm{mL}$ in the first well, and further dilution series were obtained by a 2-fold dilution (as 50, 25, and $12.5 \mu \mathrm{g} / \mathrm{mL}$ ) of the stock.

The CCK-8 assay, in brief, was performed by adding the substrate, $10 \mu \mathrm{L}$ WST-8 (2-(2-methoxy4-nitrophenyl)-3-(4-nitrophenyl)-5-(2,4-disulfophenyl)-2H-tetrazolium, monosodium salt), to $100 \mu \mathrm{L}$ cell media/well. The plates were incubated at $37^{\circ} \mathrm{C}, 5 \% \mathrm{CO}_{2}$ for $2 \mathrm{~h}$, after which the absorbance was measured. During incubation, the substrate was reduced by the enzymes in active cells to an orange formazan dye directly proportional to the number of living cells. To avoid interference, the media were aspirated and new fresh media were added just before the initiation of the assay.

NucGreen ${ }^{\circledR}$ is a cell-impermeant stain that emits bright green $(535 \mathrm{~nm})$ fluorescence when bound to DNA. Cells that have lost plasma membrane integrity are stained within minutes, making NucGreen ${ }^{\circledR}$ a stain used to estimate live/dead cell ratios. For the NucGreen viability assay, 2 drops of NucGreen reagent were added per $\mathrm{mL}$ of medium and after $5 \mathrm{~min}$ incubation, the fluorescence signal was detected at $535 \mathrm{~nm}$, with an excitation wavelength of $485 \mathrm{~nm}$ [20].

\subsection{XFCT Phantom Experiments}

The performance of the synthesized NPs as contrast agents in small-animal XFCT was investigated in an in situ imaging setting. A pipette tip (Eppendorf epTIPS, 2-200 $\mu \mathrm{L}$ ) was used as an imaging target by filling it with a Rh-PVP NPs sample dispersion in DI water at a concentration of $250 \mu \mathrm{g} / \mathrm{mL} \mathrm{Rh}$. The pipette tip was then surgically inserted in a sacrificed mouse for another study (15-week-old, female, NOD.Cg-Prkdcscid Il2rgtm1Sug/JicTac, Taconic Biosciences, Lille Skensved, Denmark). The mouse was positioned inside a $60 \mathrm{~mL}$ tube and imaged using our in-house preclinical XFCT arrangement [21]. The choice of the pipette tip as an imaging target allowed, due to its conical shape with a decreasing cross-sectional diameter, an investigation of the smallest observable feature size at the fixed NP concentration. The inner diameter of the pipette tip ranged from 0.5 to $4 \mathrm{~mm}$ along its length of $35 \mathrm{~mm}$.

Simultaneous XFCT and computed tomography (CT) was performed on the sacrificed mouse by acquiring 30 projection images over 180 degrees. Each projection image was acquired with steps of $200 \mu \mathrm{m}$ and exposure time of $10 \mathrm{~ms}$, resulting in a total scan time of $\sim 1.5 \mathrm{~min}$ for each axial slice (of $200 \mu \mathrm{m}$ thickness). We note that the chosen acquisition settings and the radiation dose, which is estimated to be $\sim 25 \mathrm{mGy}$, have been demonstrated suitable for in vivo imaging in our recent study [21]. The acquired CT data were reconstructed using a standard filtered back-projection algorithm, while the acquired XFCT data were reconstructed using an in-house developed iterative algorithm. For more details on the imaging arrangement, see Ref. [21].

\section{Results and Discussion}

Formation of metallic NPs in polyol synthesis is rather straightforward, where metal ions are reduced by the polyalcohol solvent, in our case EG. PVP acts as a pooling agent, by forming reservoirs of metal ions. In this respect, its concentration can play an important role on the size of nucleated NPs. Parameters such as reaction temperature during nucleation and crystal growth, reaction time, solvent type, and concentration of precursors and capping agents can affect the shape of final particles. In this study, we investigated the effect of different additives (PVP, KBr, CTAB, CTAC, Na-Ac, and Na-Cit) on 
the NP morphology while keeping the other parameters unaltered. Details of the prepared samples are presented in Table 1.

\subsection{Morphology Analysis}

When all the others synthesis parameters of the reaction are kept the same, the morphological variations of NPs are dependent on the type of additives used with the anionic ligands inducing the strongest morphology variation in the synthesis of NPs. For metals with a face-centered cubic (fcc) crystal structure (including Rh), the surface energy $(\gamma)$ follows the trend $\gamma(111)<\gamma(100)<$ $\gamma(110)$ with the highest surface energy in the (110) facet. These relative energies can be modified by surface-adsorbing species exposing facets that would not normally be thermodynamically favored, introducing a degree of anisotropy into the NP geometry [22].

As expected, the morphology of the obtained Rh NPs varies greatly dependent on the additives present during the synthesis (Table 1, Figure 1). With only PVP added, predominantly triangular NPs are observed (Figure 1a), revealing anisotropy due to the adsorption of PVP on the NP surface. Br-containing additives, KBr (Figure 1b) and CTAB (Figure 1d), resulted in cubic NPs. Among the carboxyl group-containing additives, acetate (NaAc, Figure 1c) and citrate (NaCit, Figure 1e) ions led to the formation of spherical NPs, which is indicative of isotropic growth, eliminating the effect of PVP. Rh NPs synthesized by the addition of CTAC (Figure 1f) display an irregular mixed polygonic particle morphology. $\mathrm{Cl}^{-}$ions did not show the same effect as the one observed in the presence of $\mathrm{Br}^{-}$ ions. The resultant NPs present a mixed polygon morphology, including trigonal, similar to the ones synthesized in the presence of PVP only. The mixed polygon morphology may be due to a complex formation between the additional $\mathrm{Cl}^{-}$ions and $\mathrm{Rh}$, influencing the NP growth dynamics [23].
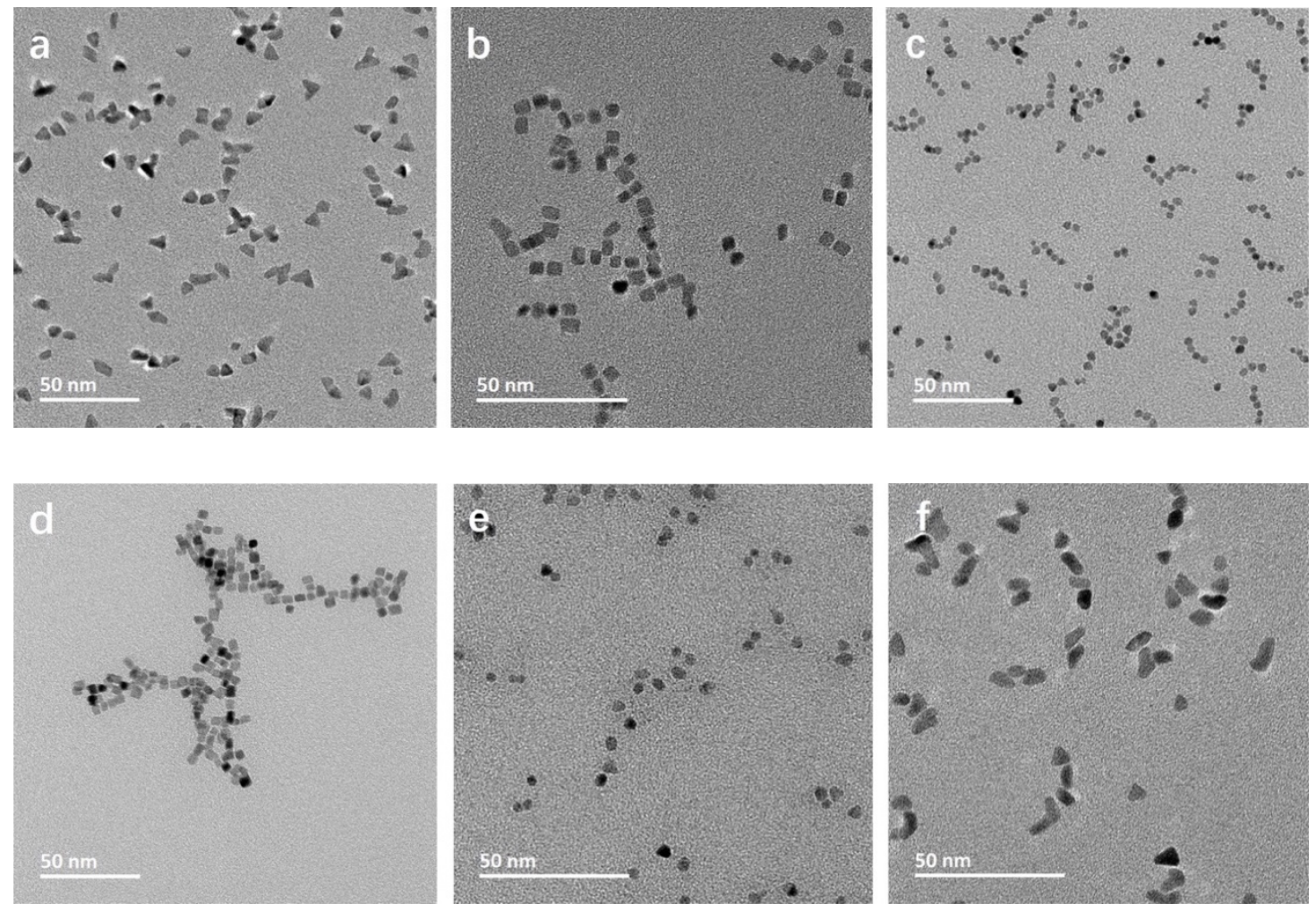

Figure 1. TEM micrographs of (a) Rh_PVP; (b) Rh_PVP-KBr; (c) Rh_PVP-Na-Ac; (d) Rh_PVP-CTAB; (e) Rh_PVP-Cit; and (f) Rh_PVP-CTAC.

The observed morphological differences are related to the preferential adsorption of anions on certain facets with the NP growth allowed predominantly along them. One common feature observed in the HRTEM micrographs of the samples (Figure 2) is the fact that the NPs obtained in the presence 
of all studied anions are single crystalline, with the measured d values matching the fcc Rh (ICDD PDF card no: 03-065-2866). Lattice fringes of Rh samples synthesized in the presence of PVP (Figure 2a), Na-Ac (Figure 2c), Na-Cit (Figure 2e), and CTAC (Figure 2f) have a d value of $0.22 \mathrm{~nm}$, which matches well with the (111) plane of fcc-Rh. The additives containing $\mathrm{Br}^{-}$ions, however, showed a slightly different passivating behavior. Br- ions are reported to passivate the (100) plane in Rh nanocrystals [14]. Our observations confirmed the stabilization of the (100) surface when $\mathrm{Br}^{-}$-bearing $\mathrm{KBr}$ (Figure 2b) and CTAB (Figure 2d) are used as additives. In both cases, the NPs have a cubic morphology with lattice fringes of $0.19 \mathrm{~nm}$ corresponding to the (200) plane of $\mathrm{Rh}$, proving that $\mathrm{Br}^{-}$ions are capable of getting adsorbed on the surface of the NPs formed, even in the presence of PVP.
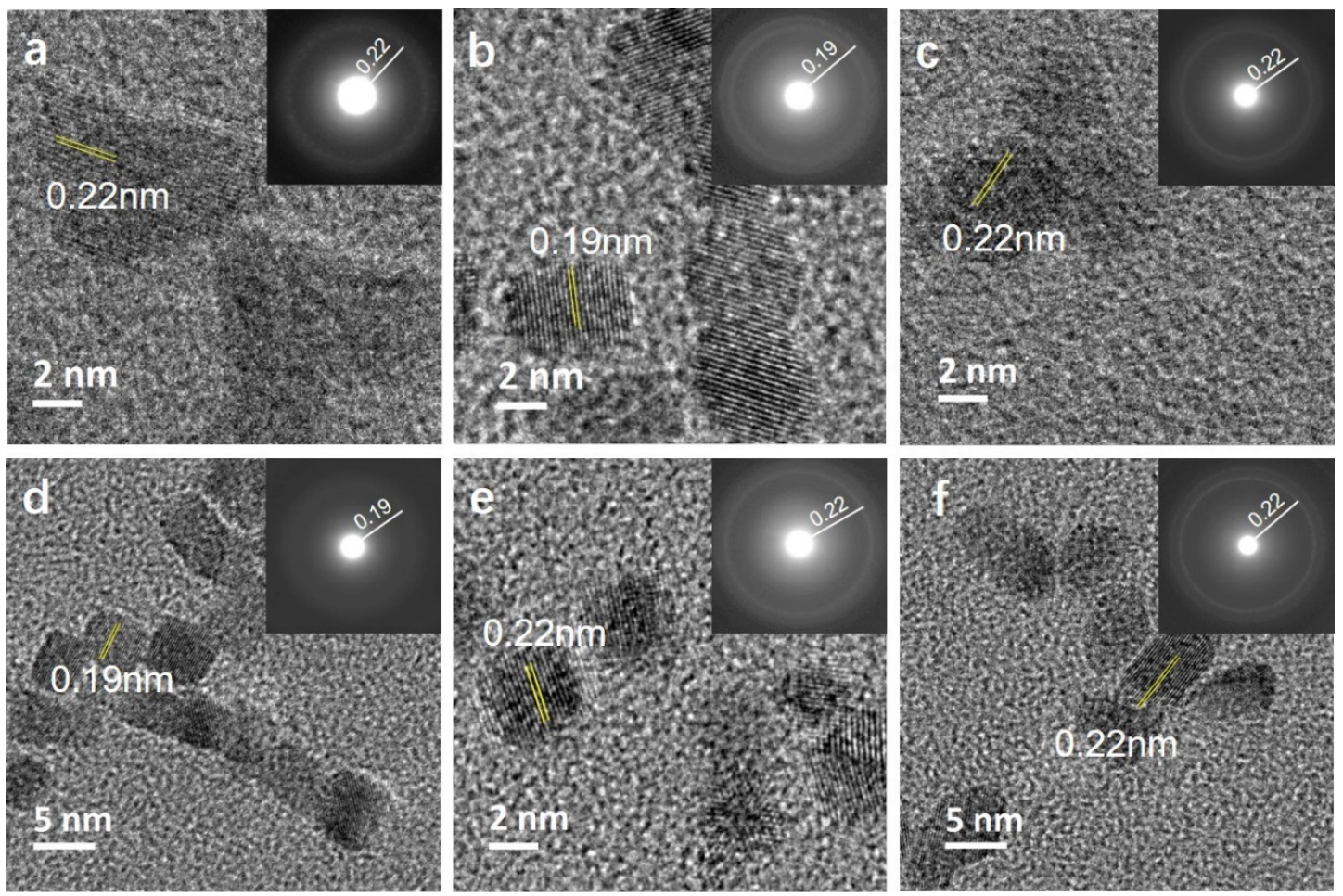

Figure 2. (a-f) HRTEM micrographs of Rh NPs: (a) Rh_PVP; (b) Rh_PVP-KBr; (c) Rh_PVP-Na-Ac; (d) Rh_PVP-CTAB; (e) Rh_PVP-Cit; and (f) Rh_PVP-CTAC (the lattice spacings are indexed for fcc Rh using ICDD PDF card no: 03-065-2866).

Plasmonic NPs of Ag and Au show a size- and morphology-dependent UV-Vis absorption behavior [24]. In order to study the effect of morphology on Rh NP absorption characteristics, we performed UV-Vis analysis on the synthesized samples. UV-Vis absorption spectra indicated that the maximum absorption wavelength due to the surface plasmon resonance (SPR) of Rh varied with particles morphology, with a peak value in the range of 210-220 nm (Figure S1). The intensity of SPR absorption is the highest for Rh NPs with a cubic morphology (Rh_PVP-KBr and Rh_PVP-CTAB), with an absorption edge at $225 \mathrm{~nm}$. A similar trend of absorption intensity and edge was observed for triangular NPs (Rh_PVP). Spherical NPs (Rh_PVP-Ac and Rh_PVP-Cit) display an absorption maximum at $210 \mathrm{~nm}$, with an absorption edge at about $220 \mathrm{~nm}$. All these samples showed a weak, broad absorption at the 270-275 nm region. Polygonic Rh NPs (Rh_PVP-CTAC) showed fluctuating absorption in the range of 200-225 nm, followed by a broad absorption centered at $275 \mathrm{~nm}$, with a very high background absorption. This may be due to the polydispersity in the shapes of NPs (observed in Figure 1f) increasing the background absorption significantly. 


\subsection{Particle Size and Surface Chemistry Analyses}

Besides the morphological effects on the Rh NPs, the dry particle size obtained from TEM micrographs, hydrodynamic size, and surface properties are also influenced by the additives used (Table 2, Figure 3). The largest NPs were formed when PVP only $(7.6 \mathrm{~nm})$ and PVP-CTAC $(6.6 \mathrm{~nm})$ are used. Bromide-containing additives, $\mathrm{KBr}$ and $\mathrm{CTAB}$, formed NPs with an average size of 4.7-4.8 nm. Carboxylic acid functionality-bearing additives, Na-Ac and Na-Cit, resulted in NPs with an average size of 3.1-3.4 $\mathrm{nm}$. The differences in NP size are ascribed to the fact that the additives clearly affect the crystal nucleation and growth kinetics in addition to directing the morphology. It may be reasonable to relate this to the notion that acetate and citrate ions allowed a fast nucleation, i.e., burst nucleation, and bromide ions constrained the growth moderately, whereas PVP only and chloride ions yielded the largest size of Rh NPs due to the impeded kinetics allowing particle ripening.

Table 2. Dry size (TEM size \pm standard deviation (SD)), hydrodynamic size (DLS size, the polydispersity index (PDI)), and zeta potential of Rh NPs with different morphologies in DIW and DMEM with $10 \%$ FBS.

\begin{tabular}{cccccc}
\hline & & \multicolumn{2}{c}{ In DIW } & \multicolumn{2}{c}{ In CCM + 10\%FBS } \\
\hline Sample & $\begin{array}{c}\text { TEM Size; Mean } \\
\pm \text { SD }(\mathbf{n m})\end{array}$ & $\begin{array}{c}\text { DLS Size } \\
\text { [PDI] }(\mathbf{n m})\end{array}$ & $\begin{array}{c}\text { Zeta Potential } \\
\mathbf{( m V )}\end{array}$ & $\begin{array}{c}\text { DLS Size } \\
\text { [PDI] (nm) }\end{array}$ & $\begin{array}{c}\text { Zeta Potential } \\
\mathbf{( m V )}\end{array}$ \\
\hline Rh-PVP & $7.6 \pm 1.6$ & $29.1[0.23]$ & 0.18 & $18.1[0.49]$ & -9.72 \\
Rh-PVP-KBr & $4.7 \pm 0.8$ & $41.1[0.23]$ & -7.74 & $16.5[0.46]$ & -11.24 \\
Rh-PVP-CTAB & $4.8 \pm 0.7$ & $97.1[0.19]$ & -11.57 & $37.8[0.64]$ & -8.76 \\
Rh-PVP-CTAC & $6.6 \pm 1.3$ & $46.2[0.39]$ & -3.59 & $19.7[0.55]$ & -5.69 \\
Rh-PVP-NaAc & $3.4 \pm 0.7$ & $31.9[0.41]$ & -9.43 & $17.8[0.44]$ & -10.02 \\
Rh-PVP-NaCit & $3.1 \pm 0.6$ & $28.3[0.18]$ & -5.05 & $50.7[0.68]$ & -9.38 \\
\hline
\end{tabular}

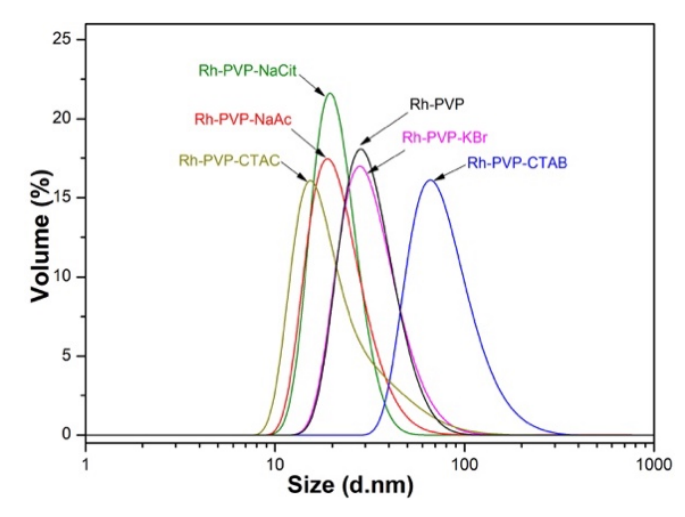

(a)

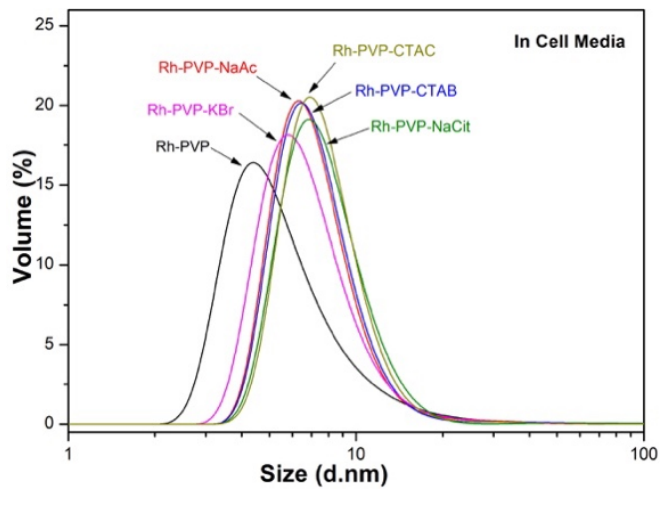

(b)

Figure 3. Hydrodynamic size distribution plots, using the DLS technique, of as-synthesized Rh NPs, with different morphologies in (a) DIW and (b) DMEM. Data presented in volume distribution.

The NPs' dispersed sizes, i.e., the hydrodynamic size, were evaluated in DIW and cell culture media-DMEM, and the results are presented graphically in Figure 3 and summarized in Table 2. Rh NPs synthesized with PVP, PVP-Cit, and PVP-Ac have an average hydrodynamic size of around $30 \mathrm{~nm}$, the NPs synthesized in the presence of PVP-KBr and PVP-CTAC have an intermediate size of $40-50 \mathrm{~nm}$ and the size of about $100 \mathrm{~nm}$ was found when PVP-CTAB was used. The polydispersity index (PDI), a parameter that defines the goodness of size distribution, where a value less than 0.3 relates to a homogeneous population [25], is lower than 0.3 for all samples except the PVP-CTAC and PVP-Ac samples. As a general trend, all the Rh NPs synthesized in this work present a very low surface charge (Table 2), under $( \pm) 30 \mathrm{mV}$. Despite the low charge density on their surface, the NPs are stable in solution probably due to the steric hindrance exerted by the polymeric chains attached to the surface. Rh_PVP NPs show near-zero surface charge at neutral $\mathrm{pH}$. A common trend for all other samples is 
the net negative surface charge on the NPs. This correlates well with the morphological differences being controlled by the adsorbed anions on the NPs. It is important to note that the size distributions of all synthesized Rh NPs, regardless of the shape, are suitable for use in XFCT bio-imaging.

Particle size distribution is also measured upon dispersing the NPs in DMEM, containing $10 \%$ FBS. Results indicate a smaller dispersed size, except Rh_PVP-Cit, with an increase in the polydispersity index to the 0.4-0.6 level. The ionic strengths of DMEM could have helped the decrease initially observed in NP clusters, while various proteins in DMEM led to larger agglomerate formations, which are detected when scattering intensity is used for hydrodynamic NP size distribution (see ESI, Figure S2). Adsorption of proteins is also influential in the final surface charge of Rh NPs in DMEM, which are observed to have enhanced their negative charge as compared to their surface charge in DIW (Table 2).

The FT-IR spectra reveal that the surface of all Rh NPs shows dominantly PVP features with no strong indication of the presence of secondary additives (Figure 4), probably due to the easy removal of these additives during the washing steps. A common feature is the shift of the position of the $\mathrm{C}=\mathrm{O}$ absorption band from 1662 for the pure PVP to around $1650 \mathrm{~cm}^{-1}$ for all NPs synthesized, indicating the conjugation of PVP via the carbonyl group onto the Rh NPs' surface. The quantitative determination of the organic material proportion in the samples by thermal gravimetric analysis (TGA) showed that the degradation of the organics was completed at $500{ }^{\circ} \mathrm{C}$ (ESI, Figure S3). The thermograms and a table summarizing the findings are presented in Figure S3. The Rh_PVP system showed the highest $\mathrm{NP}$ content of about $13 \mathrm{wt} \%$, where the NP content decreased to $6 \mathrm{wt} \%$, in a non-monotonic way for the other samples.

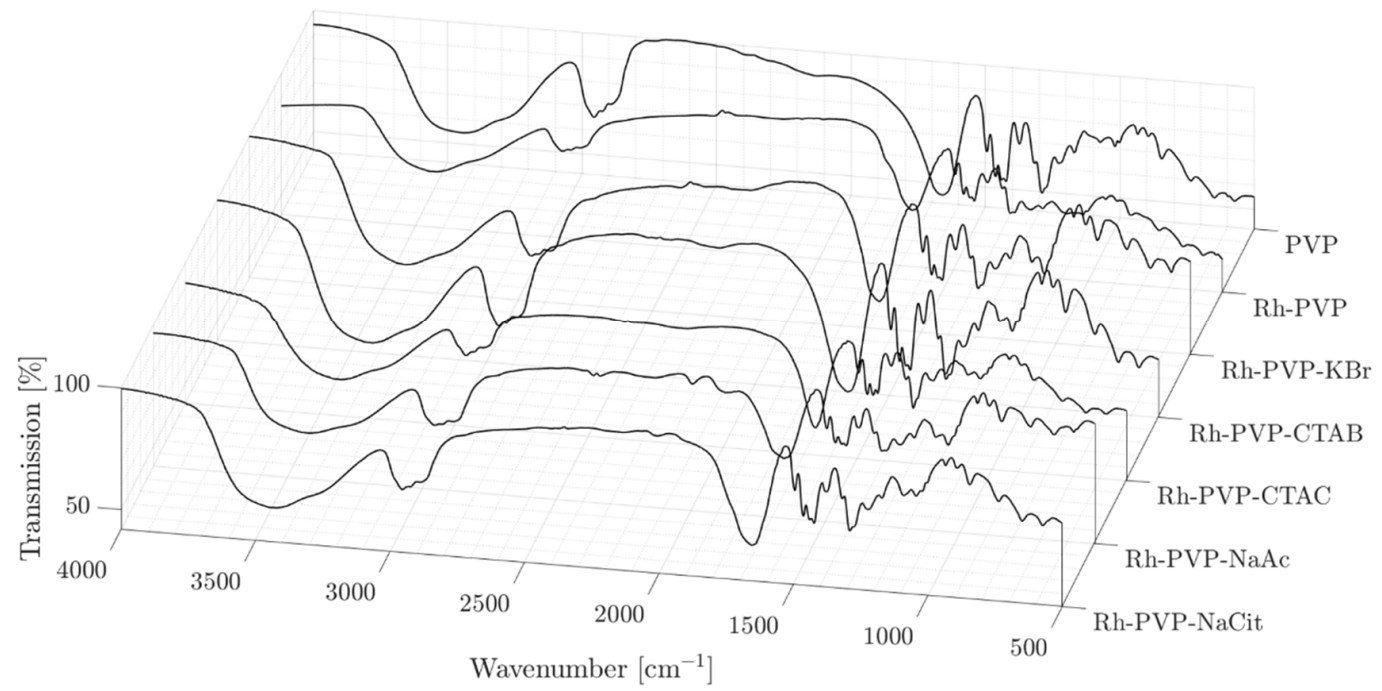

Figure 4. FT-IR spectra of pure PVP and Rh NPs; Rh_PVP, Rh_PVP-KBr, Rh_PVP-CTAB, Rh_PVP-CTAC, Rh_PVP-Ac, and Rh_PVP-Cit.

\subsection{Cytotoxicity Studies}

The cellular viability as an indicator for the toxicity potency of an agent can be affected in various ways. We, therefore, tested the membrane integrity (fluorescence NucGreen assay) and cellular metabolic activity (colorimetric CCK-8 assay) of cells exposed to Rh NPs with different morphologies in a concentration-dependent manner, with the highest dose of $250 \mu \mathrm{g} / \mathrm{mL}$ and the lowest nominal dose of $30 \mu \mathrm{g} / \mathrm{mL}$ for the 24-h exposure time. It is known that different cell types will react in a distinct way in the presence of external stimuli. Therefore, in this work the cytotoxicity of Rh NPs was evaluated on murine macrophages (RAW264.7) and a human ovarian cancer (SKOV-3) cell line. The RAW264.7 macrophages serve as a model for immune cells, while SKOV-3 is used as a model of tumoral cells to investigate if the exposure to NPs will induce a different level of toxicity in different cell lines. The half 
maximal inhibitory concentration (IC50) is specified for NPs, whenever relevant, in various toxicity assays against different cell lines.

NucGreen is staining only the cells that have lost plasma membrane integrity. The membrane integrity of RAW 264.7 is not compromised when exposed to trigonal Rh NPs (Rh_PVP). However, it is significantly compromised when exposed to cubic NPs (Rh_PVP-KBr and Rh_PVP-CTAB), at all doses. In the case of spherical NPs (Rh_PVP-NaAc (IC50: $118 \mu \mathrm{g} / \mathrm{mL}$ ) and Rh_PVP-NaCit (IC50: $120 \mu \mathrm{g} / \mathrm{mL}$ )), the membrane integrity is affected in a concentration-dependent manner, the viability increasing with the decreasing concentration of NPs. Cell viability is significantly reduced for doses $\geq 125 \mu \mathrm{g} / \mathrm{mL}$ (ESI, Figure S4). Rh NPs with a mixed polygon morphology (Rh_PVP-CTAC), presented separately in Figure S6, due to their lower concentration reaching a maximum of $100 \mu \mathrm{g} / \mathrm{mL}$, showed high viability at all the doses tested.

The exposure to triangular Rh NPs (Rh_PVP) of the RAW264.7 cell line in doses up till $125 \mu \mathrm{g} / \mathrm{mL}$ did not significantly decrease the cells' metabolic activity, with the viability reducing to about $50 \%$ at a dose of $250 \mu \mathrm{g} / \mathrm{mL}$ (Figure 5. Among the spherical NPs, Rh-Cit NPs (IC50: $56 \mu \mathrm{g} / \mathrm{mL}$ ) reached viability beyond $60 \%$ when the dose was reduced to $60 \mu \mathrm{g} / \mathrm{mL}$, while Rh-Ac NPs (IC50: $86 \mu \mathrm{g} / \mathrm{mL}$ ) showed viability of $>70 \%$ at the lowest dose of $30 \mu \mathrm{g} / \mathrm{mL}$. Cubic NPs (Rh_PVP-KBr and Rh_PVP-CTAB) showed very low viability, or a high level of toxicity, at all the doses tested. NPs with a mixed polygon morphology (Rh-CTAC, Figure 6) showed a high level of toxicity in the RAW264.7 cell line.

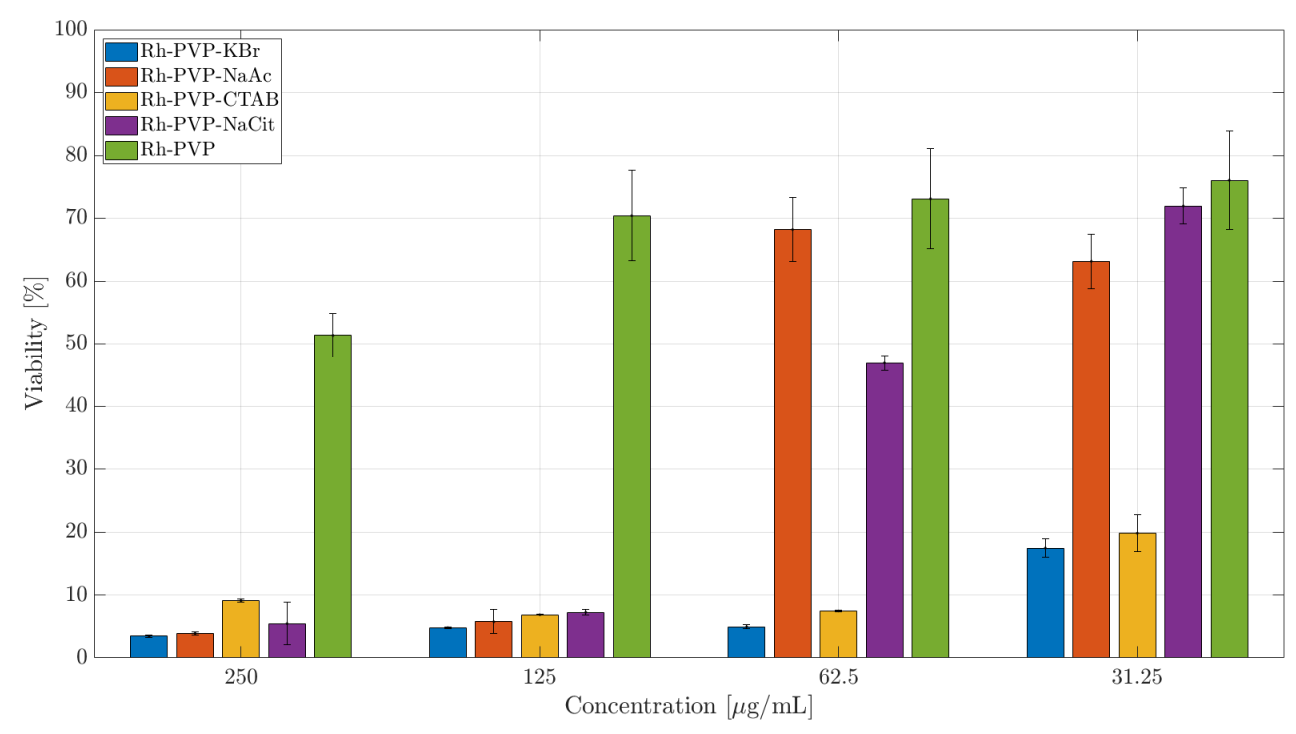

Figure 5. CCK-8 toxicity assay of Rh-NPs in the RAW 264.7 cell line after $24 \mathrm{~h}$ of incubation. The percentage of cell viability is calculated relative to the cells incubated in the absence of NPs (negative control) with $100 \%$ viability.

The cytotoxicity of some organic surface-coating agents used for NP synthesis has been studied in the literature, where the cationic surfactant $C T A B$ was reported to be highly toxic [26]. In the case of Rh NPs synthesized in the presence of CTAB and CTAC, the residue of the cationic part could cause the observed negative effect. The common character of Rh NPs synthesized in the presence of $\mathrm{KBr}$ and $\mathrm{CTAB}$ is their cubic morphology, due to surface-adsorbed $\mathrm{Br}$ - ions, which resulted in high cytotoxicity levels. $\mathrm{Br}^{-}$ions are known to exist in humans and animals and can reach up to almost half of the $\mathrm{Cl}^{-}$content in red blood cells. [27] Adsorbed inorganic $\mathrm{Br}^{-}$is, therefore, not expected to show the observed cytotoxicity in the case of $\mathrm{KBr}$-assisted synthesis. Therefore, it is reasonable to ascribe the observed cytotoxicity to the cubic NP morphology. In general, SKOV-3 cells are more resistant to external agents. Indeed, the membrane integrity of SKOV-3, maintained at $>80 \%$, is not compromised significantly (Figure S5), when exposed to trigonal (Rh_PVP) and spherical NPs (Rh_PVP-NaAc and Rh_PVP-NaCit). However, in the presence of NPs with a cubic morphology (Rh_PVP-KBr (IC50: 
$119 \mu \mathrm{g} / \mathrm{mL}$ ) and Rh_PVP-CTAB (IC50: $115 \mu \mathrm{g} / \mathrm{mL}$ )) in doses $\geq 125 \mu \mathrm{g} / \mathrm{mL}$, the viability is significantly reduced. NPs with a mixed polygon morphology (Rh_PVP-CTAC) (Figure S6) showed high viability at all the doses tested, similar to the response of macrophages.

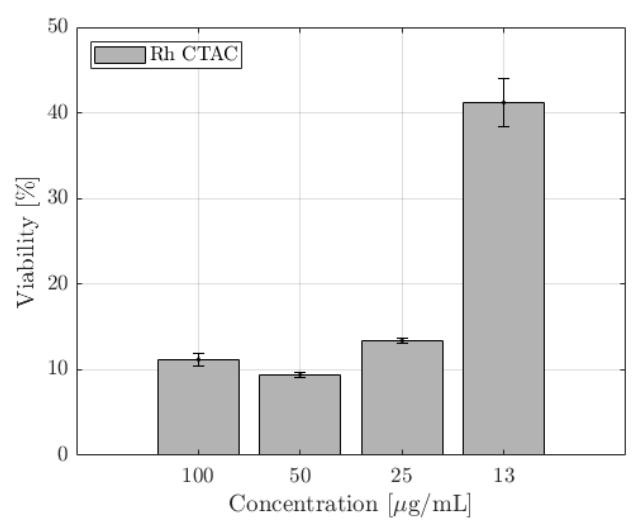

(a)

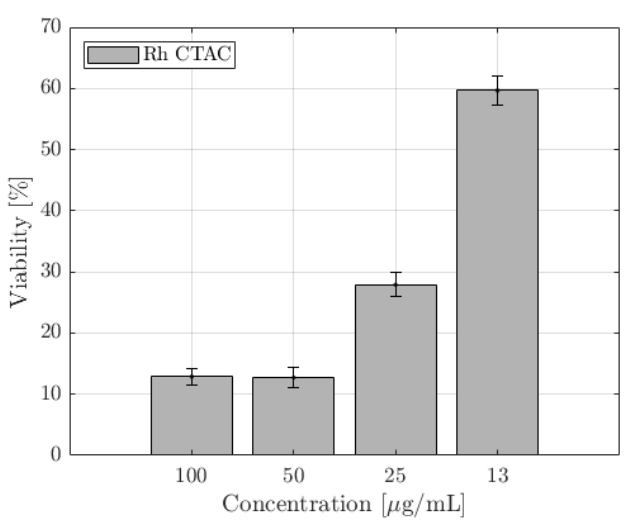

(b)

Figure 6. CCK-8 toxicity assays of Rh-CTAC NPs in the (a) RAW 264.7 and (b) SKOV-3 cell lines after $24 \mathrm{~h}$ of incubation. The percentage of cell viability is calculated taking negative control cells incubated in the absence of NPs with $100 \%$ viability.

A similar trend is observed in the CCK-8 assay (Figure 7) with the trigonal NPs (Rh-PVP) exerting the lowest toxicity followed by spherical NPs (RhPVP-NaCit and Rh-PVP-NaAc (IC50: $218 \mu \mathrm{g} / \mathrm{mL}$ )). The highest toxicity level is observed when the cells were incubated with cubic NPs (Rh_PVP-CTAB (IC50: $42 \mu \mathrm{g} / \mathrm{mL}$ ) and Rh_PVP-KBr (IC50: $41 \mu \mathrm{g} / \mathrm{mL}$ )). NPs with a mixed polygon morphology (Rh_PVP-CTAC) (Figure 6b) showed a high level of toxicity in the SKOV-3 cell line at higher doses, reaching a viability of $>60 \%$ at the lowest dose of $13 \mu \mathrm{g} / \mathrm{mL} \mathrm{NP}$ concentration.

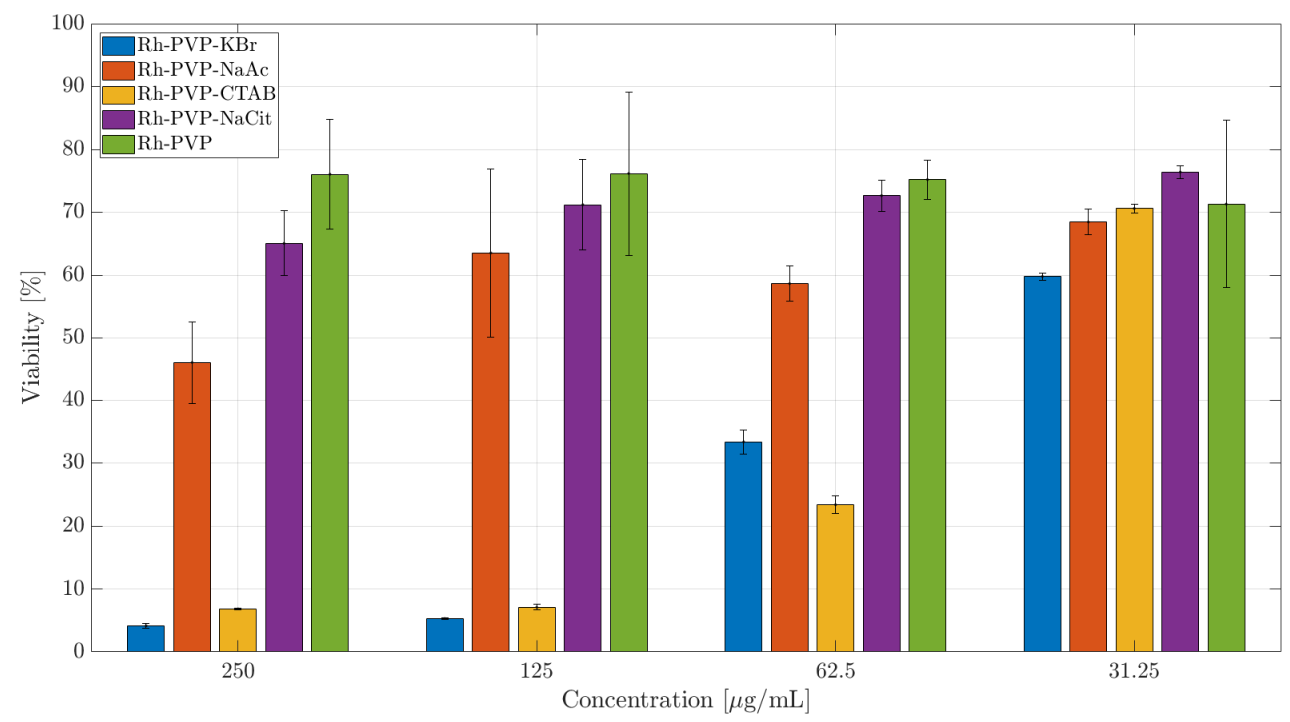

Figure 7. CCK-8 toxicity assay of Rh NPs in the SKOV-3 cell line after $24 \mathrm{~h}$ of incubation. The percentage of cell viability is calculated relative to the cells incubated in the absence of NPs (negative control) with $100 \%$ viability.

The differentiated cytotoxicity response of the macrophage cell line (RAW 264.7) and cancer cell line (SKOV-3) in the presence of the NPs might be explained by the different functions the macrophages and cancer cells are specialized to perform. While the macrophages should react to any external or internal aggression factors, the cancer cells are programed to adapt to any conditions in order to 
survive and multiply. This work showed that membrane integrity and metabolic activity, expressed as viability, are influenced to a similar extent, for both the cell lines, with respect to the morphology of Rh NPs. Particles with a mixed polygon morphology showed the highest negative impact on cellular activity, followed by cubic and spherical NPs. Trigonal NPs showed the lowest negative effect, or the highest viability, among the NP series. From the FT-IR analysis, we see no significant evidence of the presence of functional groups, especially for surfactants CTAB and CTAC. Trace amounts of these functional groups may still reside on the surface dominating PVP, or at the immediate NP surface. By looking at the significantly different response of cellular activity towards these NPs, it is reasonable to ascribe the observed effects to the NP morphology.

Similar observations were reported for noble metal NPs. For instance, gold nanorods prepared using $\mathrm{CTAB}$ were shown to be highly toxic to human skin cells due to the presence CTAB, not the gold nanorods themselves [28]. It was later shown that over-coating gold nanorods with polymers substantially reduced their cytotoxicity [29]. If any of the Rh NP morphologies showing a high level of toxicity prove to be more interesting candidates for biomedical applications, their surface chemistry can be further modified using polymers or other inorganic coatings, such as silica, to improve their biocompatibility, without adversely influencing the diagnostics function.

\subsection{XFCT Performance}

Figure 8 shows the in situ small-animal XFCT imaging experiment. Using the pipette tip (Figure 8a) as an imaging target allowed the investigation of the smallest observable feature size at the fixed NP concentration, due to its conical shape with a decreasing cross-sectional diameter. The content of the pipette tip could not be separated and visualized in the CT reconstruction, while it could be clearly visualized in the XFCT reconstruction (c.f., Figure 8b). For diameters of the pipette tip between 4 and $2.5 \mathrm{~mm}$, the NP content could be reliably reconstructed, while for diameters smaller than $2.5 \mathrm{~mm}$, it became increasingly difficult for the reconstruction algorithms to separate the XRF signal from background X-ray scattering. Below the $2 \mathrm{~mm}$ diameter mark, the reconstruction algorithms could no longer separate the XRF signal from the background. These results agree with previous simulations, which indicated that a local NP concentration of $>1 \mathrm{mg} / \mathrm{mL}$ is necessary to reach sub-mm feature visualization [30].

a

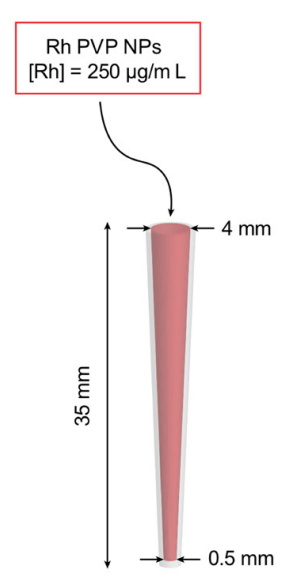

b

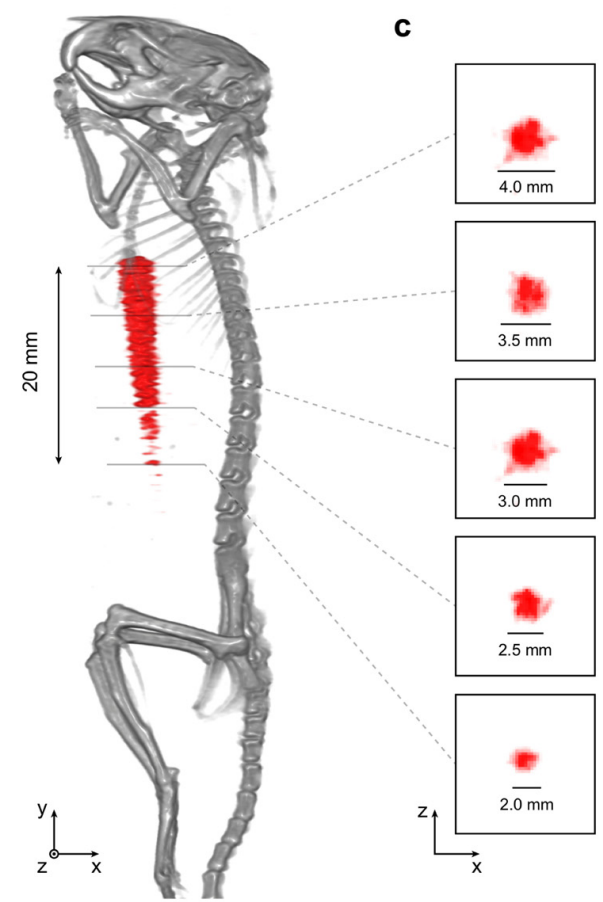

Figure 8. In situ XFCT performance of sample Rh-PVP. (a) A pipette tip was used as an imaging target 
by filling it with Rh-PVP NPs $(250 \mu \mathrm{g} / \mathrm{mL})$, where the conical shape offered a target with an inner diameter ranging from 4 to $0.5 \mathrm{~mm}$. (b) Reconstructed tomographic data visualized in 3D (grayscale: CT, red: XFCT). The portion of the pipette tip visible in the XFCT reconstruction was estimated to be $\sim 20 \mathrm{~mm}$. (c) Selected axial slices with $5 \mathrm{~mm}$ separation in y of the XFCT reconstruction, with the theoretical expected diameters at each location denoted with scale bars and numbers. Each pixel in the axial slices corresponds to $200 \times 200 \mu \mathrm{m}^{2}$.

Selected cross-sections of the XFCT reconstruction are shown in Figure 8c and compared to the theoretical inner diameters of the pipette tip at the selected locations. We note that estimated cross-sectional pipette tip diameters in the XFCT reconstruction are in the range of theoretical values between 4 and $2 \mathrm{~mm}$, and conclude, therefore, that few-mm features with a local NP concentration in the $100 \mu \mathrm{g} / \mathrm{mL}$ range can be visualized using XFCT. In other words, this type of in situ imaging experiment offers a controlled environment to investigate relations between parameters such as feature size and observable local Rh NP concentration, which is not directly possible in in vivo scenarios.

\section{Conclusions}

$\mathrm{Rh}$ nanoparticles (NPs) with various morphologies were synthesized in the presence of a large palette of additives during the polyol synthesis process. The halide salts and surfactant additives including sodium acetate, sodium citrate, $\mathrm{KBr}, \mathrm{CTAB}$, and CTAC, in addition to PVP, showed a tuning effect on the shape of the Rh NPs' morphology. When PVP was used as the only additive, trigonal NPs were obtained. Additives with $\mathrm{Br}^{-}$ions (CTAB and $\mathrm{KBr}$ ) resulted in NPs with a cubic morphology, while those with carboxyl groups (sodium citrate and acetate) formed spheroid $\mathrm{NPs}^{-\mathrm{Cl}^{-}}$ions (CTAC) resulted in a mixture of polygon morphologies. The observed cytotoxicity response evaluated using two different viability assays, on macrophages and ovarian cancer cell lines, shows a strong dependence on the NPs morphology. The cells exposed to trigonal Rh NPs showed the highest viability, among the NP series. Particles with a mixed polygon morphology showed the highest cytotoxicity, followed by cubic and spherical NPs, which can be correlated with a more shape-dependent response triggering. The performance of Rh NPs as contrast agents for small-animal XFCT was demonstrated in an in situ experiment, showing promise for future in vivo imaging. This work provides a detailed route for the synthesis, morphology control, and characterization of Rh NPs as viable contrast agents for XFCT bio-imaging.

Supplementary Materials: The following are available online at http://www.mdpi.com/2079-4991/10/11/2129/s1, Figure S1: UV-Vis absorption spectra of Rh NPs; Rh_PVP, Rh_PVP-KBr, Rh_PVP-Ac, Rh_PVP-CTAB, Rh_PVP-Cit, and Rh_PVP-CTAC. Figure S2: Hydrodynamic particle size distribution plots, using DLS, of as-synthesized Rh NPs, with different morphologies. Data presented in intensity distribution to show the agglomerates formed. Figure S3: TGA thermograms of Rh NPs; Rh_PVP, Rh_PVP-KBr, Rh_PVP-CTAB, Rh_PVP-CTAC, Rh_PVP-Ac, and Rh_PVP-Cit. Figure S4. NucGreen toxicity assay of Rh NPs in the RAW 264.7 cell line after $24 \mathrm{~h}$ of incubation. The percentage of cell viability is calculated relative to the cells incubated in the absence of NPs (negative control) with $100 \%$ viability. Figure S5. NucGreen toxicity assay of Rh NPs in the SKOV-3 cell line after 24 h of incubation. The percentage of cell viability is calculated relative to the cells incubated in the absence of NPs (negative control) with $100 \%$ viability. Figure S6. NucGreen toxicity assays of Rh-CTAC NPs in (a) RAW 264.7 and (b) SKOV-3 cell lines after $24 \mathrm{~h}$ of incubation. The percentage of cell viability is calculated taking negative control cells incubated in the absence of NPs with $100 \%$ viability.

Author Contributions: M.S.T. and H.M.H. conceived of the presented ideas. Conceptualization and methodology, Y.L., K.S., M.S., B.B. and M.S.T.; validation, M.S., C.V. and K.S.; formal analysis, Y.L., G.M.S.; writing-original draft preparation, Y.L.; writing-review and editing, all. M.S.T. and H.M.H. supervised the work. All authors have read and agreed to the published version of the manuscript.

Funding: This research was funded by the Wallenberg Foundation. Y.L. acknowledges the support from the Chinese Scholarship Council (CSC).

Acknowledgments: We thank Torbjörn Gräslund and Haozhong Ding (KTH Biotechnology) for fruitful discussions. 
Conflicts of Interest: The authors declare no conflict of interest. The funders had no role in the design of the study; in the collection, analyses, or interpretation of data; in the writing of the manuscript, or in the decision to publish the results.

\section{References}

1. Xu, L.; Liu, D.; Chen, D.; Liu, H.; Yang, J. Size and shape controlled synthesis of rhodium nanoparticles. Heliyon 2019, 5, e01165. [CrossRef] [PubMed]

2. Lee, S.R.; Vara, M.; Hood, Z.D.; Zhao, M.; Gilroy, K.D.; Chi, M.; Xia, Y. Rhodium Decahedral Nanocrystals: Facile Synthesis, Mechanistic Insights, and Experimental Controls. ChemNanoMat 2018, 4, 66-70. [CrossRef]

3. Xie, S.; Liu, X.Y.; Xia, Y. Shape-controlled syntheses of rhodium nanocrystals for the enhancement of their catalytic properties. Nano Res. 2015, 8, 82-96. [CrossRef]

4. Cao, D.X.; Wieckowski, A.; Inukai, J.; Alonso-Vante, N. Oxygen reduction reaction on ruthenium and rhodium nanoparticles modified with selenium and sulfur. J. Electrochem. Soc. 2006, 153, A869-A874. [CrossRef]

5. Zhou, M.; Wang, H.; Vara, M.; Hood, Z.D.; Luo, M.; Yang, T.H.; Bao, S.; Chi, M.; Xiao, P.; Zhang, Y.; et al. Quantitative Analysis of the Reduction Kinetics Responsible for the One-Pot Synthesis of Pd-Pt Bimetallic Nanocrystals with Different Structures. J. Am. Chem. Soc. 2016, 138, 12263-12270. [CrossRef] [PubMed]

6. Xia, Y.; Xia, X.; Peng, H.C. Shape-Controlled Synthesis of Colloidal Metal Nanocrystals: Thermodynamic versus Kinetic Products. J. Am. Chem. Soc. 2015, 137, 7947-7966. [CrossRef]

7. Ganguly, P.; Breen, A.; Pillai, S.C. Toxicity of Nanomaterials: Exposure, Pathways, Assessment, and Recent Advances. ACS Biomater. Sci. Eng. 2018, 4, 2237-2275. [CrossRef]

8. Lewinski, N.; Colvin, V.; Drezek, R. Cytotoxicity of nanoparticles. Small 2008, 4, 26-49. [CrossRef]

9. Buzea, C.; Pacheco, I.I.; Robbie, K. Nanomaterials and nanoparticles: Sources and toxicity. Biointerphases 2007, 2, MR17-MR71. [CrossRef]

10. Nel, A.; Xia, T.; Mädler, L.; Li, N. Toxic Potential of Materials at the Nanolevel. Science 2006, $311,622$. [CrossRef]

11. Alkilany, A.M.; Murphy, C.J. Toxicity and cellular uptake of gold nanoparticles: What we have learned so far? J. Nanopart. Res. 2010, 12, 2313-2333. [CrossRef] [PubMed]

12. Kang, S.; Shin, W.; Choi, M.-H.; Ahn, M.; Kim, Y.-K.; Kim, S.; Min, D.-H.; Jang, H. Morphology-Controlled Synthesis of Rhodium Nanoparticles for Cancer Phototherapy. ACS Nano 2018, 12, 6997-7008. [CrossRef] [PubMed]

13. Yu, N.F.; Tian, N.; Zhou, Z.Y.; Huang, L.; Xiao, J.; Wen, Y.H.; Sun, S.G. Electrochemical Synthesis of Tetrahexahedral Rhodium Nanocrystals with Extraordinarily High Surface Energy and High Electrocatalytic Activity. Angew. Chem.-Int. Ed. 2014, 53, 5097-5101. [CrossRef]

14. Zhang, H.; Li, W.; Jin, M.; Zeng, J.; Yu, T.; Yang, D.; Xia, Y. Controlling the Morphology of Rhodium Nanocrystals by Manipulating the Growth Kinetics with a Syringe Pump. Nano Lett. 2011, 11, 898-903. [CrossRef] [PubMed]

15. Biacchi, A.J.; Schaak, R.E. The solvent matters: Kinetic versus thermodynamic shape control in the polyol synthesis of rhodium nanoparticles. ACS Nano 2011, 5, 8089-8099. [CrossRef]

16. Li, Y.; Shaker, K.; Larsson, J.C.; Vogt, C.; Hertz, H.M.; Toprak, M.S. A Library of Potential Nanoparticle Contrast Agents for X-Ray Fluorescence Tomography Bioimaging. Contrast Media Mol. Imaging 2018, 2018. [CrossRef] [PubMed]

17. Li, Y.; Shaker, K.; Svenda, M.; Vogt, C.; Hertz, M.H.; Toprak, S.M. Synthesis and Cytotoxicity Studies on Ru and Rh Nanoparticles as Potential X-Ray Fluorescence Computed Tomography (XFCT) Contrast Agents. Nanomaterials 2020, 10, 310. [CrossRef]

18. Dobrovolskaia, M.A.; Patri, A.K.; Zheng, J.; Clogston, J.D.; Ayub, N.; Aggarwal, P.; Neun, B.W.; Hall, J.B.; McNeil, S.E. Interaction of Colloidal Gold Nanoparticles with Human Blood: Effects on Particle Size and Analysis of Plasma Protein Binding Profiles. Nanomed. Nanotechnol. Biol. Med. 2009, 5, 106-117. [CrossRef]

19. FDA. Guidance for Industry: Pyrogen and Endotoxins Testing: Questions and Answers; FDA: Rockville, MD, USA, 2012.

20. Roth, B.L.; Poot, M.; Yue, S.T.; Millard, P.J. Bacterial Viability and Antibiotic Susceptibility Testing with SYTOX Green Nucleic Acid Stain. Appl. Environ. Microbiol. 1997, 63, 2421-2431. [CrossRef] 
21. Shaker, K.; Vogt, C.; Katsu-Jimenez, Y.; Kuiper, R.; Andersson, K.; Li, Y.; Larsson, J.; Rodriguez-Garcia, A.; Toprak, M.; Arsenian-Henriksson, M.; et al. Longitudinal In-Vivo X-Ray Fluorescence Computed Tomography with Molybdenum Nanoparticles. IEEE Trans. Med. Imaging 2020. [CrossRef]

22. Hoefelmeyer, J.D.; Niesz, K.; Somorjai, G.A.; Tilley, T.D. Radial Anisotropic Growth of Rhodium Nanoparticles. Nano Lett. 2005, 5, 435-438. [CrossRef]

23. Shlenskaya, V.I.; Efremenko, O.A.; Oleinikova, S.V.; Alimarin, I.P. Chloride Complexes of Rhodium (Iii) in Aqueous Solutions. Bull. Acad. Sci. USSR Div. Chem. Sci. 1969, 18, 1525-1527. [CrossRef]

24. Zhang, J.Z.; Noguez, C. Plasmonic Optical Properties and Applications of Metal Nanostructures. Plasmonics 2008, 3, 127-150. [CrossRef]

25. Danaei, M.; Dehghankhold, M.; Ataei, S.; Hasanzadeh Davarani, F.; Javanmard, R.; Dokhani, A.; Khorasani, S.; Mozafari, M.R. Impact of Particle Size and Polydispersity Index on the Clinical Applications of Lipidic Nanocarrier Systems. Pharmaceutics 2018, 10, 57. [CrossRef] [PubMed]

26. Zhang, Y.; Newton, B.; Lewis, E.; Fu, P.P.; Kafoury, R.; Ray, P.C.; Yu, H. Cytotoxicity of Organic Surface Coating Agents Used for Nanoparticles Synthesis and Stability. Toxicol. Vitr. 2015, 29, 762-768. [CrossRef]

27. Van Leeuwen, F.X.R.; Sangster, B.; Hildebrandt, A.G. The Toxicology of Bromide Ion. Crit. Rev. Toxicol. 1987, 18, 189-213. [CrossRef]

28. Wang, S.; Lu, W.; Tovmachenko, O.; Rai, U.S.; Yu, H.; Ray, P.C. Challenge in Understanding Size and Shape Dependent Toxicity of Gold Nanomaterials in Human Skin Keratinocytes. Chem. Phys. Lett. 2008, 463, 145-149. [CrossRef] [PubMed]

29. Alkilany, A.M.; Nagaria, P.K.; Hexel, C.R.; Shaw, T.J.; Murphy, C.J.; Wyatt, M.D. Cellular Uptake and Cytotoxicity of Gold Nanorods: Molecular Origin of Cytotoxicity and Surface Effects. Small 2009, 5, 701-708. [CrossRef]

30. Shaker, K.; Larsson, J.C.; Hertz, H.M. Quantitative Predictions in Small-Animal X-Ray Fluorescence Tomography. Biomed. Opt. Express 2019, 10, 3773. [CrossRef]

Publisher's Note: MDPI stays neutral with regard to jurisdictional claims in published maps and institutional affiliations.

(C) 2020 by the authors. Licensee MDPI, Basel, Switzerland. This article is an open access article distributed under the terms and conditions of the Creative Commons Attribution (CC BY) license (http://creativecommons.org/licenses/by/4.0/). 